Gastroenterologia 1960;93:118

\title{
Corticotropin in Acute Cholecystitis
}

\begin{tabular}{l|l|l} 
H. & Herman & Wahren
\end{tabular}

Eskilstuna, Sweden

Summary

In the surgical clinic of Eskilstuna Central Hospital we have treated cases of acute pancreatitis with ACTH for a period of three years. One year ago we also started to treat acute gall bladder affections with the same hormone.

Five graphs from our case reports were shown. A marked and immediate decrease of temperature can be seen after injection of 100 units of ACTH (“Acton prolongatum”).

It must be born in mind that the temperature has a tendency to decline suddenly in acute gall bladder affections. However, our study includes more than 40 cases where the temperature fell immediately following an injection of ACTH. It therefore seems obvious to us that a causal connection exists between the decrease in temperature and the ACTH. The underlying causes are however not yet understood.

A slight increase in the bilirubin level was noticed in most cases of acute cholecystitis. After ACTH the level decreased practically in all cases.

We have also noticed a decrease in the bilirubin level after ACTH in cases of extra hepatic obstruction. On this account we cannot recognize the value of ACTH as a differential diagnostic test between the two types of jaundice. 